\title{
Optimal Control of Drug in an HIV Immunological Model
}

\author{
${ }^{1}$ F.S Akinboro, ${ }^{2}$ S. Alao, F.O Akinpelu, B. Gbadamosi \\ ${ }^{1,2}$ Department of Pure \& Applied Mathematics, LAUTECH Ogbomoso, Nigeria.
}

\begin{abstract}
A model for the interaction of $H I V$ with the $C D 4^{+} T$ cells was examined in which an optimal treatment parameter was introduced to control the infectivity termin the HIV dynamic model. The control class was chosen to be a measurable function defined with an objective functional which maximizes the T cell count and minimizes the systemic cost based on the percentage effect of the antiretroviral therapy drug. Optimal control was characterized by applying pontryagin's maximum principle. The values of the objective function at the optimal control shows that the greatest effect do occur when treatment is initiated earliest. Also, results of the numerical simulations indicate that the rate of uninfected $\mathrm{CD}^{+} \mathrm{T}$ increased and virus population decreased due to treatment parameter.
\end{abstract}

Keywords:Drug threrapy, Immunological model, Optimal control, Objective function, Pontryagin's principle

\section{Introduction}

HIV is a lentivirus that causes Acquired Immunodeficiency Syndrome (AIDS), a condition in humans in which progressive failure of the immune system allows life-threatening opportunistic infections and cancers to thrive. Infection with HIV occurs by the transfer of blood, semen, vaginal fluidor breast milk. Within these bodily fluids, HIV is present as both free virus particles and virus within infected immune cells.

Many mathematical models have been derived in order to describe the dynamics of HIV infection in the bloodstream where cell-free-viral spread is the predominant route of viral spread. To model the influence of HIV on T-cell growth, some consideration were made by AlanPerelsonet al. [1] which take into account a number of features of the life history of the virus. However when HIV infects a cell the enzyme reverse transcriptase which it carries, makes a DNA copy of its RNA genome. The viral DNA called the provirus is then duplicated with the cell's DNA every time the cell divides. Thus, once a cell is infected it remains infected for life $[1,2,3,4,5]$.

The use of drugs to suppress replication of the HIV has transformed the face of AIDS in the developed world. Pronounced reductions in illness and death have been achieved and healthcare utilization has diminished. HIV therapy has also provided many new insights into the pathogenesis and cellular dynamics of HIV infection.In HIV antiretroviral therapy, drugs act by either blocking the integration of viral RNA into the host cells, or by inhibiting the proper cleavage of viral proteins inside an infected cell. Drugs such as AZT, DDC, DDI and D4T all work as reverse transcriptase inhibitor.

Optimal control of drug for HIV systems requires two components. The first is an understanding of the system in the absence of treatment and the second is a description of the effects of treatment $\mathrm{u}(\mathrm{t})$. A number of work has been done on drug treatment in different settings.Kirshneret al. [3] studied a mathematical model whereby the chemotherapy reduces viral production rather than viral infectivity which is more applicable to drugs such as protease inhibitors[4,5].

Rosenberget al.[7] used a mathematical modeling with control to develop structured treatment interruption strategies for HIV infection. The goal of the article suggested that mathematical models describing biological processes taking place within a patient over time can be used to design adaptive treatment strategies. Although there has been considerable progressing management of HIV infection using highly active antiretroviral therapies, continuous treatment with these agents involves significant cost and burden, toxicities, development of drug resistance, and problems with adherence; these latter complications are of particular concern in substance abusing individuals.

Nejadet al. [2] presented a fast solution for solving HIV-infection dynamics and chemotherapy optimization based on fuzzy. Two ordinary differential equations systems which models interaction between HIV viruses and human body immune system were used. In addition, Kamyad (AVK) discretization method was introduced and used to solve the mathematical models. The results are then extended to the other domain points of the mathematical model by a fuzzy inference estimator.

Olarteet al. [8] worked on a robust control-based HIV-treatment for infected patients. The dynamics of the immune system's response to infection was modeled using system of nonlinear model with separate efficacy coefficients for protease inhibitor (PIs) and reverse transcriptase inhibitors (RTIs). Numerical simulation results showed that the control law could lead to long-term stable conditions, even in extreme cases. 
In this paper, we deal specifically with when and how treatment should be initiated assuming that treatment can only be continued for a finite time interval until drug resistance is developed. We presented an HIV dynamic immunological in which the optimal control term is the coefficient of the viral infectivity term. The benefit of the treatment is to increase the uninfected $C D 4^{+} T$ cell counts and to decrease the infected cells. We define our objective function to maximize the benefit based on $\mathrm{T}$ cell count and minimize the weight factor based on the amount of drug.Pontryagin's maximum principle is used to derive conditions on the optimal control. The optimality system is then solved numerically using a fourth orderRunge-kutta scheme. Graphical representation of numerical simulations is presented with the discussion of the result.

\section{Model Formulation}

To model these event, the population density of uninfected $C D 4^{+} T$ cells, latently infected cells, actively infected cells and free virus[1,2,6] are denoted by T,L,A,Vrespectively. where $\frac{d T}{d t}, \frac{d L}{d t}, \frac{d A}{d t}, \frac{d V}{d t}$ represent the rate of change in population densities uninfected, latently infected, actively infected $\mathrm{T}$ cells respectively at time $t$.

$$
\left\{\begin{array}{l}
\frac{d T}{d t}=\frac{s}{1+V}-\mu_{1} T-k_{1} V T+r T\left(1-\frac{T+L+A}{T_{\max }}\right) \\
\frac{d L}{d t}=k_{1} V T-\mu_{2} L-k_{2} L \\
\frac{d A}{d t}=k_{2} L-\mu_{3} A \\
\frac{d V}{d t}=N \mu_{3} A-k_{1} V T-\mu_{4} V
\end{array}\right.
$$

In the first compartment, $\frac{s}{1+V}$ is a source term from the thymus and represent the rate of generation of new $C D 4^{+} T$ cells. The $\mathrm{T}$ cells have a finite life span with a death rate $\mu_{1}$ per cell, in the second compartment latently infected $\mathrm{T}$ cells are assumed to have anatural death rate $\mu_{2}$ and the actively infected $\mathrm{T}$ cells, virus cells die at the rate $\mu_{3}, \mu_{4}$ respectively.Small letter ' $\mathrm{r}$ ' in the first compartment represent the coefficient of the growth rate of $\mathrm{T}$ cells, which is a logistic type growth which ensures that the $\mathrm{T}$ cells never grow larger than $T_{\max }$.

The term $K_{1} V T$ models the rate that free virus infects $C D 4^{+} T$ cells which implies that after an uninfected $\mathrm{T}$ cell becomes infected, it becomes latently infected $\mathrm{T}$ cell. Then the latently infected $\mathrm{T}$ cellsbecomes actively infected at the rate $K_{2}$ in the third compartment. Also, the last compartment models the free virus population in which an assumption is made that when an actively infected $C D 4^{+} T$ cells becomes stimulated by antigen exposure, replication of virus begins [9]. Further $\mathrm{N}$ viruses are formed before the host cell dies, the term $-\mu_{4} V$ takes into account loss of infectively or natural viral loss [3].

Let $T_{0}$ denote the normal steady state $\mathrm{T}$ cell population value (in the absence of virus),

$$
T_{0}=\left(\frac{-\left(\mu_{1}-r\right) T_{\text {max }}+\sqrt{\left(\left(\mu_{1}-r\right) T_{\text {max }}\right)^{2}+4 r s T_{\text {max }}}}{2 r}\right)
$$

Thus, an adequate initial condition for equation(1) are $T(0)=T_{0}, L(0)=0, A(0)=0, V(0)=0$ and for infection by free virus $T(0)=T_{0}, L(0)=L_{0}, A(0)=A_{0}, V(0)=V_{0}$. Similarly, immunological models describing the interaction of HIV infection with the immune system was studied by Denise[3] and Shelly [9]. 
TABLE 1

Parameter and Constants

$\mu_{1}=$ death rate of uninfected $C D 4^{+} T$ cell population

$0.02 d^{-1}$

$\mu_{2}=$ death rate of latently infected $C D 4^{+} T$ cell population

$0.02 d^{-1}$

$\mu_{3}=$ death rate of actively infected $C D 4^{+} T$ cell population

$0.24 d^{-1}$

$\mu_{4}=$ death rate of free virus

$2.4 d^{-1}$

$k_{1}=$ rate $T(t)$ becomes infected by free virus

$2.4 \times 10^{-5} \mathrm{~mm}^{3} \mathrm{~d}^{-1}$

$k_{2}=$ rate $L(t)$ cells convert to actively infected cells

$\mathrm{r}$ =rate of growth for the $C D 4^{+} T$ cell population

$3 \times 10^{-3} \mathrm{~mm}^{3} \mathrm{~d}^{-1}$

$0.03 d^{-1}$

$\mathrm{N}=$ number of free virus produced by $A(t)$ cells

1200

$T_{\max }=$ Maximum $C D 4^{+} T$ cell population level

$1.5 \times 10^{3} \mathrm{~mm}^{-3}$

$s=$ source term for uninfected $C D 4^{+} T$ cells

$10 d^{-1} m^{-3}$

2.1

MODEL ANALYSIS

The HIV model can be considered as being immunological and mathematically well posed if the model is reasonable in the sense that no cell population goes negative and no population grows unbounded.

Theorem 1: If $T(0), L(0), A(0), V(0)$ are non-negative , then $T(t), L(t), A(t), V(t)$ are positively invariant for all $t>0$. The non-negative orthant $R_{+}^{4}=\left\{t \varepsilon R^{4} \mid t \geq 0\right\}$ is called a positively invariant region if a trajectory that starts in the non-negative orthant remains there forever.

From equation (1), we have

$$
\begin{array}{ll}
\left.\frac{d T}{d t}\right|_{T=0}=\frac{s}{1+V} \geq 0, & \left.\frac{d L}{d t}\right|_{L=0}=K_{1} V T \geq 0 \\
\left.\frac{d A}{d t}\right|_{A=0}=K_{2} L \geq 0, & \left.\frac{d V}{d t}\right|_{V=0}=N \mu_{4} A \geq 0
\end{array}
$$

Hence the proof.

From the above theorem we conclude that the model can be considered well posed and bounded.

\section{Optimal Control Formulation}

The basic idea of control term $[5,9,10,11]$ in this work is to reduce the rate at which virus infect the uninfected $C D 4^{+} T$ cells and our control represents the percentage effect the antiretroviral drugs has on the interaction of $\mathrm{T}$ cells with the virus. The chemotherapy treatment term $\mathrm{u}(\mathrm{t})$ multiplies the parameter $k_{1} V T$ in equation (1) to give equation (3) below.

$$
\left.\begin{array}{rl}
\frac{d T}{d t} & =\frac{s}{1+V}-\mu_{1} T-u(t) k_{1} V T+r T\left(1-\frac{T+L+A}{T_{\max }}\right) \\
\frac{d L}{d t} & =u(t) k_{1} V T-\mu_{2} L-k_{2} L \\
\frac{d A}{d t} & =k_{2} L-\mu_{3} A \\
\frac{d V}{d t} & =N \mu_{3} A-u(t) k_{1} V T-\mu_{4} V
\end{array}\right\}
$$

withgiven initial condition for $\mathrm{T}(\mathrm{t}), \mathrm{L}(\mathrm{t}), \mathrm{A}(\mathrm{t}), \mathrm{V}(\mathrm{t})$ at $t=t_{0}$ where $0 \leq u(t) \leq 1$

Considering the problem that often arises in the use of drugs such as harmful side effect, as well as the ineffectiveness of treatment after a certain time due to the capability of the virus to mutate and become resistant 
to the drug. A finite time interval [tstart,tfinal] was impose since it was assumed that therapeutic period only has a limited treatment window. In clinical practice antiretroviral therapy is initiated at $t_{0}$, the time at which treatment begins and treatment last for a given period which must be less than 2 years.

$t_{\text {start }} \leq t \leq t_{\text {final }}$ implies $t_{\text {final }}-t_{\text {start }}<2$ years

Defining the objective functional, a quadratic cost was chosen since we have reason to believe that the cost function is a non-linear function of $u^{*}$. This is based on the fact that there no linear relationship between the effect of treatment on $\mathrm{T}$ cells or virus.

If the control $u(t)=0$ represents maximal use of drug, then the maximal cost is represented as $(1-u(t))^{2}$.

The objective functional is defined as

$$
J(u)=\int_{t_{\text {start }}}^{t_{\text {final }}}\left[T(t)-\frac{1}{2} B(1-u(t))^{2}\right] d t
$$

The parameter $B \geq 0$ represents the desired 'weight' on the benefit and cost.

The goal is to characterize the optimal control $u^{*}$ satisfying

$$
\max _{0 \leq u \leq 1} J(u)=J\left(u^{*}\right)
$$

Theproblem is stated as,

$$
\max _{u} \int_{0}^{t_{\text {final }}}\left[T(t)-\frac{1}{2} B\left(1-u(t)^{2}\right] d t\right.
$$

Where the set of controls $\mathrm{K}$ given by

$$
k=\left\{u:\left[0, t_{\text {final }}\right] \rightarrow[0,1]: 0 \leq u(t) \leq 1\right\}
$$

For $(T(t), L(t), A(t), V(t))$ subject to $[2,5,6]$ the state equation (3) and the conditions

$T(0)=T_{0}, L(0)=L_{0}, A(0)=A_{0}, V(0)=V_{0}$, where $T\left(t_{f}\right), L\left(t_{f}\right), A\left(t_{f}\right), V\left(t_{f}\right)$ are free.

\subsection{CHARACTERISATION OF AN OPTIMAL CONTROL}

Pontryagin's maximum principle

If $u^{*}(t)$ and $x^{*}(t)$ are optimal then there exists an adjoint variable $\lambda(t)$ such that

$$
H\left(t, x^{*}(t), u, \lambda(t)\right) \leq H\left(t, x^{*}(t), u^{*}(t), \lambda(t)\right.
$$

at each time for all $\mathrm{u}$ where the Hamiltonian is defined by

$$
\begin{aligned}
& H(t, x(t), u(t), \lambda(t))=f(t, x(t), u(t))+\lambda(t) g(t, x(t), u(t)) \\
& \lambda^{\prime}(t)=-\frac{\partial H(t, x(t), u(t), \lambda(t))}{\partial x} \quad \text { where } \lambda(T)=0
\end{aligned}
$$

Using Pontryagrin's Maximum principle to find the optimal control we first define the Hamitonian $[10,11,12]$ i.e. the integrand of the objective function coupled with right hand side of the state equation through the adjoint variables $\lambda_{1}(t), \lambda_{2}(t), \lambda_{3}(t), \lambda_{4}(t)$ with the penalty multipliers $\omega_{1}(t), \omega_{2}(t)$ attach the control constraints.

$$
\begin{aligned}
& H\left(T, L, A, V, u, \lambda_{1}(t), \lambda_{2}(t), \lambda_{3}(t), \lambda_{4}(t)\right)=T(t)-\frac{1}{2} B(1-u(t))^{2}+ \\
& \lambda_{1}\left(\frac{s}{1+v}-\mu_{1} T-u(t) k_{1} v T+r T\left(1-\frac{T+L+A}{T_{\max }}\right)\right) \\
& +\lambda_{2}\left(u(t) k_{1} v T-\mu_{2} L-k_{2} L\right)+\lambda_{3}\left(k_{2} L-\mu_{3} A\right)+\lambda_{4}\left(N \mu_{3} A-u(t) k_{1} v T-\mu_{4} V\right) \\
& +\omega_{1}(t) u(t)+\omega_{2}(t)(1-u(t)),
\end{aligned}
$$

Where $\omega_{1}(t) \geq 0, \omega_{2}(t) \geq 0$ are penalty multipliers satisfying $\omega_{1}(t) u(t)=0, \omega_{2}(t)(1-u(t))=0$

at the optimal $u^{*}$. Therefore the adjoint system is 


$$
\left.\begin{array}{l}
\lambda_{1}^{\prime}=-\frac{\partial H}{\partial T}=-\left[1+\lambda_{1}\left(-\mu_{1}+r\left(1-\frac{T+L+A}{T_{\max }}\right)-\frac{r T}{T_{\max }}-u(t) k_{1} V\right)+\lambda_{2} k_{1} V-\lambda_{4} u(t) k_{1} V\right] \\
\lambda_{2}^{\prime}=-\frac{\partial H}{\partial L}=-\left(-\lambda_{1} \frac{r T}{T_{\max }}-\lambda_{2}\left(\mu_{2}+k_{2}\right)+\lambda_{3} k_{2}\right) \\
\lambda_{3}^{\prime}=-\frac{\partial H}{\partial A}=-\left(-\lambda_{1} \frac{r T}{T_{\max }}-\lambda_{3} \mu_{3}+\lambda_{4} N \mu_{3}\right) \\
\lambda_{4}^{\prime}=-\frac{\partial H}{\partial V}=-\left[-\lambda_{1} \frac{s}{(1+V)^{2}}-\lambda_{1} u(t) k_{1} T+\lambda_{2} u(t) k_{1} T+\lambda_{4}\left(-u(t) k_{1} T-\mu_{4}\right)\right]
\end{array}\right\}
$$

where $\lambda_{1}\left(t_{\text {final }}\right)=\lambda_{2}\left(t_{\text {final }}\right)=\lambda_{3}\left(t_{\text {final }}\right)=\lambda_{4}\left(t_{\text {final }}\right)=0$

Using the optimality condition, the langrangian is maximized w.r.t. to $u$ at the optimal $u^{*}$

$$
\text { i.e }\left.\frac{\partial H}{d u}\right|_{u=u^{*}}=0
$$

The Hamiltonian can be written as

$$
\begin{aligned}
& H=-\frac{1}{2} B(1-u(t))^{2}-\lambda_{1} u(t) k_{1} V T+\lambda_{2} u(t) k_{1} V T-\lambda_{4} u(t) k_{1} V T+\omega_{1}(t) u(t) \\
& +\omega_{2}(t)(1-u(t))+\text { other terms without } u .
\end{aligned}
$$

Then, differentiating the expression for $\mathrm{H}$ with respect to $u$, we have

$$
\begin{aligned}
& \frac{\partial H}{\partial u}=B(1-u(t))-\lambda_{1} k_{1} V T+\lambda_{2} k_{1} V T-\lambda_{4} k_{1} V T+\omega_{1}(t)-\omega_{2}(t) \\
& \left.\frac{\partial H}{\partial u}\right|_{u=u^{*}}=B\left(1-u^{*}\right)-\lambda_{1} k_{1} V T+\lambda_{2} k_{1} V T-\lambda_{4} k_{1} V T+\omega_{1}(t)-\omega_{2}(t)=0 \\
& \Rightarrow B-B u^{*}-\lambda_{1} k_{1} V T+\lambda_{2} k_{1} V T-\lambda_{4} k_{1} V T+\omega_{1}(t)-\omega_{2}(t)=0 \\
& u^{*}(t)=\frac{B+\left(\lambda_{2}-\lambda_{4}-\lambda_{1}\right) k_{1} V T+\omega_{1}(t)-\omega_{2}(t)}{B}
\end{aligned}
$$

where $u^{*}$ denote the optimal control value.

To determine an expression for the optimal control (without $\omega_{1}, \omega_{2}$ ) a standard optimality[12,13]technique is utilized. We consider three cases in examining the expression of $u^{*}$ :

Case 1. On the set $\left\{t \mid 0<u^{*}(t)<1\right\}, \omega_{1}(t)=0=\omega_{2}(t)$. Hence the optimal control is $u^{*}(t)=\frac{B+\left(\lambda_{2}-\lambda_{4}-\lambda_{1}\right) k_{1} V T}{B}$

Case 2. On the set $\left\{t \mid u^{*}(t)=1\right\}, \omega_{1}(t)=0_{\text {Hence }}$

$$
1=u^{*}(t)=\frac{\left(\lambda_{2}-\lambda_{4}-\lambda_{1}\right) k_{1} V T-\omega_{2}(t)}{B}+1
$$

Which implies that $0 \leq \omega_{2}(t)=\left(\lambda_{2}-\lambda_{4}-\lambda_{1}\right) k_{1} V T$ and $1 \leq \frac{\left(\lambda_{2}-\lambda_{4}-\lambda_{1}\right) k_{1} V T+B}{B}$

Case 3. On the set $\left\{t \mid u^{*}(t)=0\right\}, \omega_{2}(t)=0$.Hence,

$$
0=u^{*}(t)=\frac{\left(\lambda_{2}-\lambda_{4}-\lambda_{1}\right) k_{1} V T+\omega_{1}(t)+B}{B}
$$

Therefore $\omega_{1}(t) \geq 0$, this implies $\frac{\left(\lambda_{2}-\lambda_{4}-\lambda_{1}\right) k_{1} V T+B}{B} \leq 0$ 
hence,

$u^{*}(t)=\left(\frac{\left(\lambda_{2}-\lambda_{4}-\lambda_{1}\right) k_{1} V T+B}{B}\right)^{+}=0$

Combining these 3 cases, the optimal control is characterized as

$u^{*}(t)=\min \left(\left(\frac{\left(\lambda_{2}-\lambda_{4}-\lambda_{1}\right) k_{1} V T+B}{B}\right)^{+}, 1\right)$

where $\left(\frac{\left(\lambda_{2}-\lambda_{4}-\lambda_{1}\right) k_{1} V T+B}{B}\right)^{+}= \begin{cases}\left(\frac{\left(\lambda_{2}-\lambda_{4}-\lambda_{1}\right) k_{1} V T}{B}+1\right) & \text { if }\left(\lambda_{2}-\lambda_{4}-\lambda_{1}\right) k_{1} V T+B>0 \\ 0 & \text { if }\left(\lambda_{2}-\lambda_{4}-\lambda_{1}\right) k_{1} V T+B \leq 0\end{cases}$

The state and adjoint differential equation together with the optimal control characterization $[12,13,14,15]$ are solved numerically to illustrate and control the result. Since our state and adjoint equations have conditions at $t_{\text {final }}$, an iterative program can be created to numerically simulate solutions.

\section{Numerical Results and Discussion}

The optimal control is obtained by solving theoptimality system consisting of the state system and adjoint variable using an iterative method. We start to solve the state equation with a guess controls over the simulated time using Runge-Kutta scheme of order four. Because of the transversality conditions, the adjoint equation are solved by a backward fourth-order Runge-Kutta scheme using current iterations solution of the state equation and the iteration continues until convergence. This process is repeated and iterations stopped if the values of the unknowns at the previous iterations are very close to the ones at the present iterations.

In order to find the optimal solution, we need initial values for uninfected $\mathrm{T}$ cells, latently infected $\mathrm{T}$ cells, actively infected cells and the virus population. Equation (1) is then solved with initial condition $L_{0}=0, A_{0}=0, V_{0}=0$. With this parameters, the number of uninfected $C D 4^{+} T$ cells $T_{0}$ is stabilized around $1000 \mathrm{~mm}^{-3}$ which is less than $T_{\max }$ and this number correspond with the natural average number of $\mathrm{T}$ cells in a millimiter cube of blood.

In this section, we investigate numerically the immunological [9] model without optimal control term and also the effect of the optimal control term with varying weight factor ' $\mathrm{B}$ ' in equation (3). Using the parameter and constant in Table1 with initial conditions $T_{0}=1000, L_{0}=0, A_{0}=0, V_{0}=1$ which is an interpretation of early infection by the virus in a system, we determine the effect on the $C D 4^{+} T$ cell count in each compartment of the system.

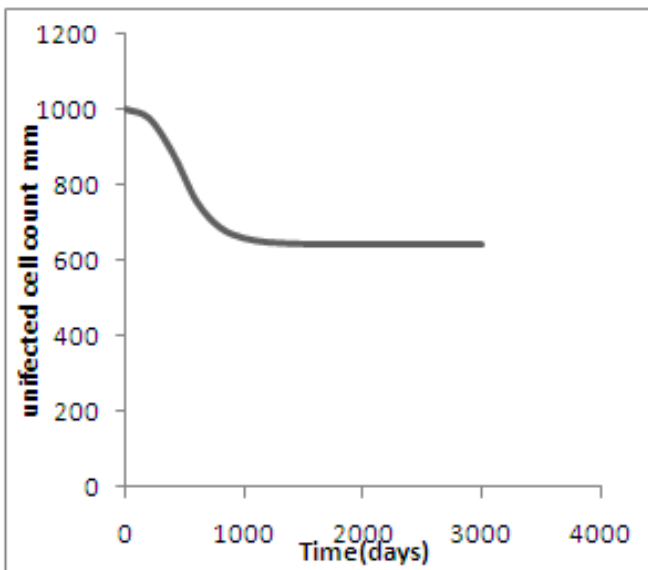

Fig. 1(a)

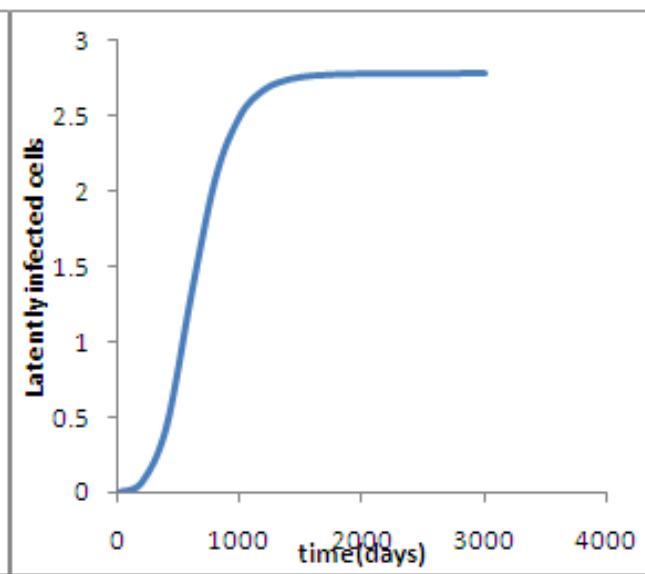

Fig. 1(b) 


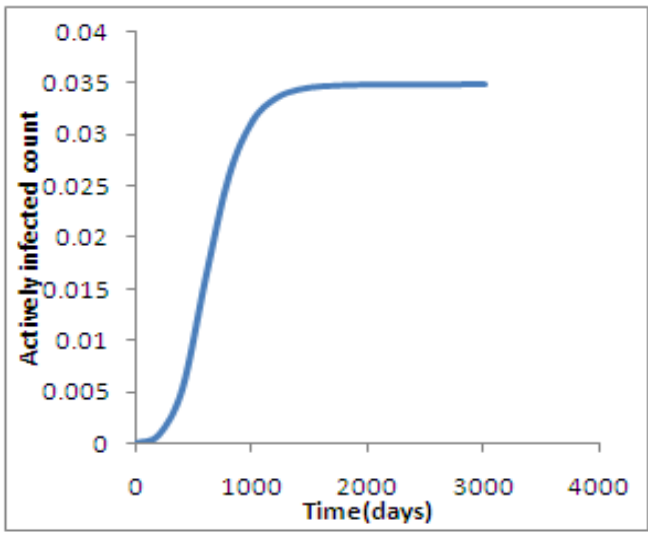

Fig. 1(c)

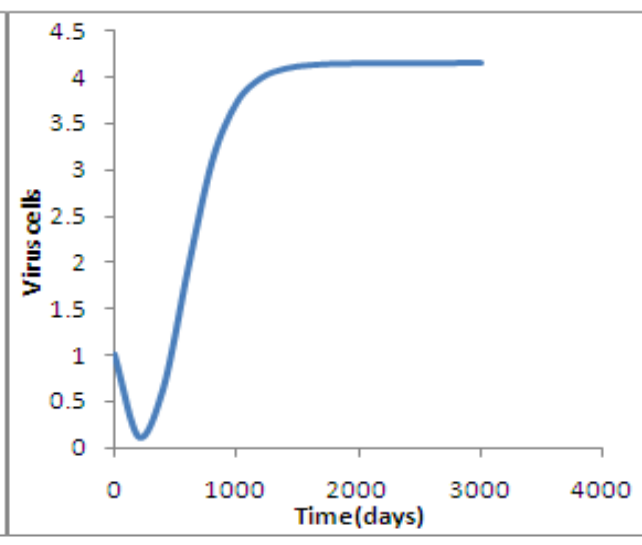

Fig. 1(d)

Figure 1.The graph shows the behavior of each compartment after the onset of infection without any medical treatment:

In actual sense the chemotherapy for an infected patient must be less than 2 years, we assume that the HIV antiretroviral therapy period is 360 days. To illustrate the effect of optimal control parameter [2] in the immunological model, we consider an infected patient with $T_{0}=400.99, L_{0}=2 A_{0}=0.2 V_{0}=4, h=1$ and iterate the numerical scheme for 360 days.



Fig 2(a)

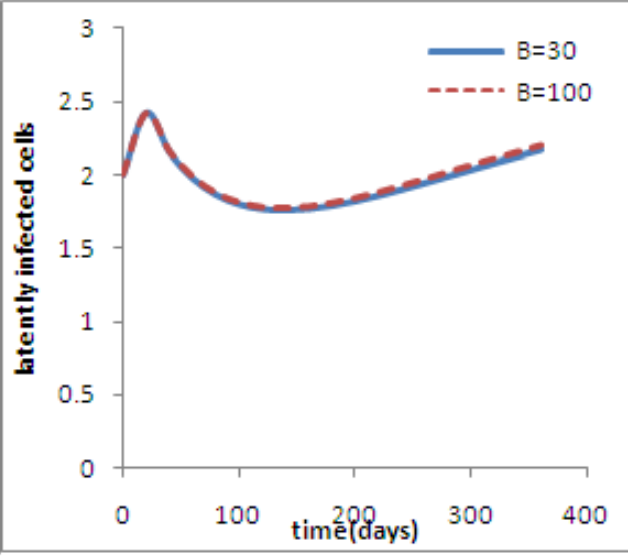

Fig 2(b)

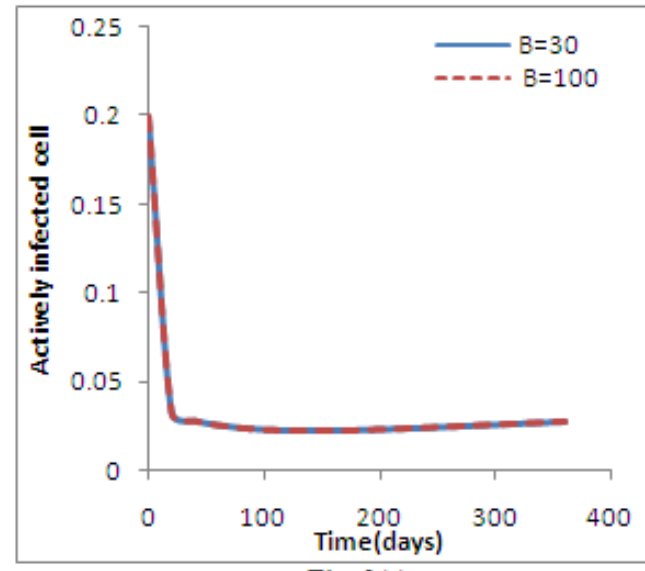

Fig. 2(c)



Fig. 2(d)

Figure 2. The graph shows the immune system dynamics in contact with HIV during the treatment period.

Fig. 2(a-d) shows that increase in weight factor $B=30$ to $B=100$ which is inversely related to the percentage of drug given. For the higher weight factor the optimal drug given produces a lower $\mathrm{T}$ cell concentration and a higher virus concentration compared to a lower weight factor. 
Also, considering $\mathrm{B}=30$ as a case study it wasobserved in fig. 2(a)that due to the optimal control term the uninfected T cells population increasesfrom $400 \mathrm{~mm}^{-3} \mathrm{~T}$ cell to $681 \mathrm{~mm}^{-3} \mathrm{~T}$ cells at time $\mathrm{t}=200$ but later decreased to $674 \mathrm{~mm}^{-3} \mathrm{~T}$ cells. The decrease is due to the fact that the immunological virus developed strains resistance to the drug after 200 days of treatment which is a signal for treatment to stop. Fig. 2(b)Show that latently infected cells population for the treated system decreases and later increases with time due to virus mutation. Similarly, Fig.2(c)\& 2(d) show that actively infected cells and the viral load both decreases which implies the viral load can be control to a very low level.

Table 2:The Valueof the Objective function at theOptimal Control $\mathrm{u}^{*}$.

\begin{tabular}{|l|l|l|}
\hline Days after onset of infection & To cell count & Objective function value J \\
\hline 300 & $941.99 \mathrm{~mm}^{-3}$ & 383,175 \\
\hline 500 & $814.88 \mathrm{~mm}^{-3}$ & 357,346 \\
\hline 1000 & $659.60 \mathrm{~mm}^{-3}$ & 337,408 \\
\hline
\end{tabular}

In table 2 above, it was observed that the objective functional when treatment is initiated 300 days after the onset of the infection is greater than when initiated at 500 days after the onset of the infection. This revealed that early treatment of the infection is optimal.

\section{Conclusion}

In this work, antiretroviral therapy was optimally controlled considering a four compartmental HIV model. We use the pontryagin's maximum to determine optimal dynamic control and then solved numerically to get the optimal control value. It was discovered that for higher weight factor, the optimal treatment parameter produces a lower $\mathrm{T}$ cell concentration and a higher virus concentration compared to a lower weight factor.

Also, comparing the value of the objective function $J(u)$ at the optimal control $u^{*}$, it was observe that the greatest effect of treatment thus occur when treatment is initiated earliest when $\mathrm{T}$ cell counts are highest, after the onset of infection.

The result of the numerical simulation indicates that the rate of uninfected $C D 4^{+} T$ increased and virus population decreased due to treatment parameter $\mathrm{u}(\mathrm{t})$.

\section{References}

[1]. S. Alan and W. Patrick,Mathematical Analysis of HIV-1 Dynamics in Vivo-Society, Industrial and Applied Mathematics, Vol. 41, No. 1,1999, pp 3-44.

[2]. H. Ghiasi and N. Chahkandi, Presentation of a Fast Solution For Solving HIV-Infection Dynamics and chemotherapy optimization based on fuzzy AVK Method, Journal of AIDS and HIV Research Vol4(3),2012, pp.60-67.

[3]. D. Kirschner, S. Lenhart and S. Serbin,Optimal control of the chemotherapy of HIV, Journal of Mathematical Biology,1997,pp 35 $775-792$.

[4]. D.Kirschner and G.F. Webb,A model for Treatment Strategy in the chemotherapy of AIDS, Bullettin of Mathematical Biology. 1996.

[5]. H. Zarei, A.V Kamyad and S. Effati, Multiobjective Optimal control of HIV Dynamics, Hindawi publishing corporation, Mathematical problem in Engineering, 2010, pp.1-29.

[6]. A. Heydari, M.H. Farahiand A.A Heydari, Optimizing Chemotherapy in an HIV Model by a Pair of Optimal Control. International Journal of Applied Mathematics vol.18.2006, No. 4. PP 389-402.

[7]. E.S. Rosenberg, M.Davidian and H. Thomas, Using mathematical modeling and control to develop structured treatment interruption strategies for HIV infection.Drug Alcohol Depend. May 2007. 88(suppl 2):S41-S51.

[8]. E.A.Olarte, C.Ramirez and H. Morales: Designing robust control-based HIV treatment. RevistaIngenieria E invastigacionvol 28 No. 2, AGOSTO DE $2008(80-88)$.

[9]. R.Fister, S.Lenhart and S.M. Joseph, Optimizing Chemotherapy in an HIV Model, Electronic Journal of Differential Equation Vol 32,1998, pp 1-12.

[10]. H. Behncke,Optimal control of deterministic epidemics, Optimal Control Appl. Meth., vol21, 2000pp. $269-285$.

[11]. F.H. Clarke, The maximum principle in optimal control, J. of Cybernetics and Control,2005, pp.709-722.

[12]. F. Clarke, The Pontryagin Maximum Principle and a Unified Theory of Dynamic Optimization, Pontryagin Centennial Conference, 2008, pp. 1-20.

[13]. H.R. Joshi, Optimal Control of an HIV Immunology Model, Optimal. Control Appl. Meth.2002,vol 23 pp. $199-213$.

[14]. H. R. Joshi, S. Lenhart, M. Li and L. Wang, Optimal Control Methods Applied to Disease Models, AMS proceeding volume on Emerging Diseases, Contemporary Mathematics 410, 2006, pp. 187-207.

[15]. R. Miller and S.Lenhart, An Introduction to Optimal Control with an Application in Disease Modeling, DIMACS Series in Discrete Mathematics Volume 75,2010, pp.61-81. 